\title{
Integral Field Spectroscopy of the center of the Draco Dwarf Spheroidal
}

\author{
John R. Jardel ${ }^{*}{ }^{a}$ Karl Gebhardt,${ }^{a}$ Maximilian Fabricius, ${ }^{b}$ and Niv Drory ${ }^{c}$ \\ ${ }^{a}$ The University of Texas \\ 1 University Station C1400, Austin, TX 78712 \\ ${ }^{b}$ Max-Planck Institut für extraterrestrische Physik \\ Giessenbachstrasse, D-85741 Garching bei München, Germany \\ ${ }^{c}$ Instituto de Astronomía, Universidad Nacional Autónoma de México \\ Avenida Universidad 3000, Ciudad Universitaria, C.P. 04510 México D.F., México \\ Email: jardeleastro.as.utexas.edu
}

\begin{abstract}
We use the VIRUS-W Integral Field Spectrograph to simultaneously obtain radial velocities for 17 stars in the central $105^{\prime \prime} \times 55^{\prime \prime}$ of the Draco dwarf spheroidal (dSph) galaxy. We apply a colormagnitude diagram filter to these stars which leaves 12 probable Draco members. From these 12 member stars we reconstruct a Line-of-Sight Velocity Distribution (LOSVD) and calculate a velocity dispersion of $\sigma=16.1_{-5.5}^{+0.8} \mathrm{~km} \mathrm{~s}^{-1}$. This LOSVD will allow us to dynamically model the center of the galaxy on smaller spatial scales where we hope to determine the slope of the dark matter density profile as well as investigate the possibility of an intermediate-mass black hole.
\end{abstract}

Frank N. Bash Symposium New Horizons In Astronomy,

October 9-11, 2011

Austin Texas

${ }^{*}$ Speaker. 


\section{Introduction}

The centers of dwarf spheroidal galaxies (dSphs) are interesting tests for the cold dark matter (CDM) model of structure formation. Since they are some of the most dark matter dominated galaxies discovered, they are believed to be relatively unaffected by the gravity of the stellar component. This makes the determination of their dark matter density profiles more straightforward than in massive galaxies, and we can compare to CDM simulations more directly. Currently, observers are finding dark matter profiles with a flat central core (e.g. [1];[8]) while CDM simulations predict all galaxies should have a cuspy central profile ([7]).

Obviously it is important to have kinematics that sample the inner regions of these galaxies so that observations are able to differentiate between cored and cuspy dark halos. Since the Local Group dSphs are relatively nearby, we are able to resolve individual stars and target them for spectroscopy. Multi-fiber spectroscopy (MFS) would appear to be ideally suited to this task as it allows for the simultaneous targeting of many individual stars. Indeed [12] provide the best data set available by using this technique (over 5,000 stars in four dSphs). However, one of the limitations of MFS is that it is difficult to position multiple fibers closely together. Thus, the centers of these dSphs are frequently under-sampled.

Where MFS becomes difficult, spectroscopy with Integral Field Units (IFUs) can be useful. IFUs often have a closely-packed array of fibers, and this high density of fibers allows for simultaneous observation of many stars in a dense field. The drawback to IFUs, however, is that fiber positions are fixed.

We present here observations of the central region of Draco using integral field spectroscopy. Our data are the most centrally-located velocities available for Draco. We analyze these data and calculate the line-of-sight velocity distribution (LOSVD) of stars within the central $10 \mathrm{pc}$ of Draco. We plan to use these data to further constrain the slope of the dark matter density profile through orbit-based dynamical models in a later paper.

\section{Observations}

We use the VIRUS-W IFU ([2]) on the 2.7m Harlan J. Smith Telescope at McDonald Observatory to target the center of the Draco dSph. This IFU packs 267 fibers into its $105^{\prime \prime} \times 55^{\prime \prime}$ field of view with a $1 / 3$ filling factor. Each fiber is $3{ }^{\prime \prime} 2$ in diameter on the sky. We observed in the spectral range covering roughly $4900 \AA$ to $5500 \AA$ at a resolving power of $R=6800$. This region allows radial velocities to be extracted from the $\mathrm{Mg} b$ stellar absorption feature.

Our observations were taken over the first half of five nights between 2011 August 1-5. Conditions were excellent for the first four nights with good transparency, and seeing was never worse than $2^{\prime \prime}$. We took the standard array of bias, twilight flat, and arc lamp calibration images. Since most of the fibers in the IFU are on empty sky, we did not need separate sky exposures. Our science exposures consist of 57 15-minute integrations, which we later combine. Total exposure time is roughly 14 hours.

Figure 1 shows the location of the IFU overlaid on a Hubble Space Telescope (HST) image from [9]. Note that typical seeing at McDonald Observatory is $2^{\prime \prime}$, much larger than the HST 


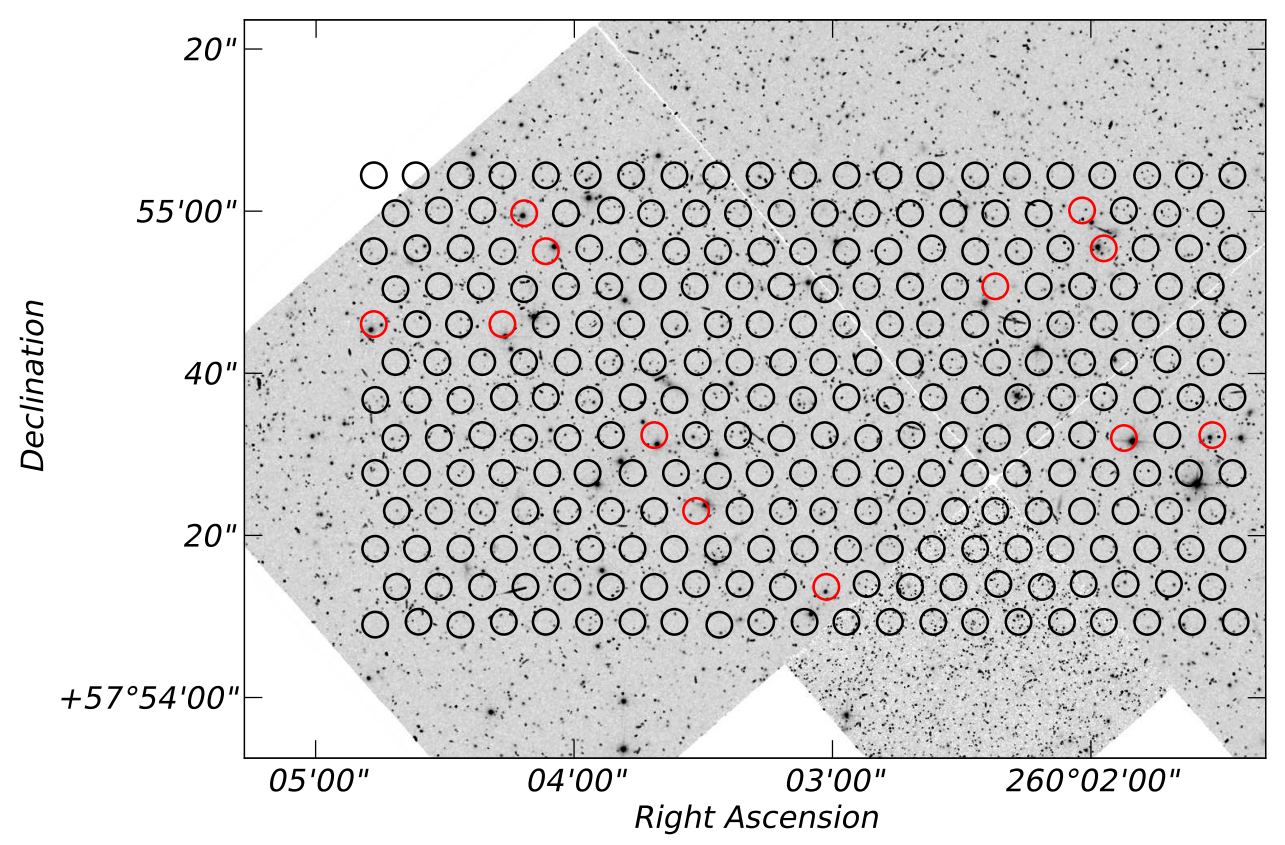

Figure 1: VIRUS-W IFU overlaid on top of an HST image from [9]. Red circles highlight fibers containing stars used in the analysis.

point-spread function. Red circles denote fibers with stars later used in the kinematic analysis. We obtained useful spectra of 17 stars with apparent magnitudes $V<21$.

\section{Analysis}

To extract radial velocities we use the brightest star with known velocity in the field as our velocity standard. Using the IRAF task FXCOR we cross-correlate the other 16 spectra to this standard. Since this star's heliocentric velocity is known and it is in the same field of view, we do not need to further correct for heliocentric motion.

To determine membership, we place the 17 observed stars in a color-magnitude diagram (Figure 2). Stars that lie away from the red giant branch are discarded as non-members, as are stars with velocity offsets greater than $50 \mathrm{~km} \mathrm{~s}^{-1}$ from systemic velocity $V_{\text {sys }}$. We keep all 12 stars marked as red asterisks in Figure 2. Note that blind sigma-clipping of the velocities also results in these same 12 stars being classified as members.

We plan to study the mass profile of Draco with dynamical models (e.g.[4];[11];[5]). In order to use these 12 stars in future models we must first reconstruct the line-of-sight velocity distribution (LOSVD) of the center of the galaxy. The LOSVD is essentially a smoothed histogram of line-ofsight radial velocities for stars in a given region of the galaxy. We calculate this by using an adaptive kernel density estimate, adapted from [10] and explained in detail in [3]. We are able to estimate the $1-\sigma$ uncertainty in the LOSVD through bootstrap resamplings of the velocity data. 


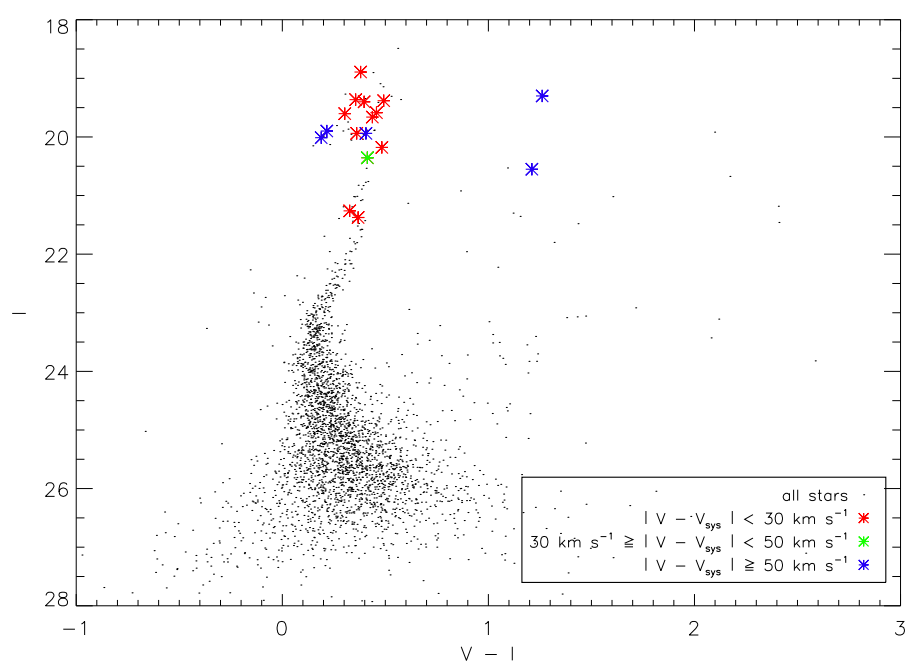

Figure 2: Color-magnitude diagram of Draco from HST photometry ([9]). Colored asterisks are stars we observe, coded according to their offset from Draco's systemic velocity $V_{\text {sys }}$.

\section{Results}

Figure 3 shows the LOSVD we calculate for the center of Draco. The stars in this LOSVD are orbiting at projected radii between 2 and $20 \mathrm{pc}$. We can fit Gauss-Hermite moments to the LOSVD and measure the mean velocity $V=3.8_{-7.3}^{+3.4} \mathrm{~km} \mathrm{~s}^{-1}$ and velocity dispersion $\sigma=16.1_{-5.5}^{+0.8} \mathrm{~km} \mathrm{~s}^{-1}$. The higher order moments are consistent with zero. This LOSVD will be an important kinematic constraint in future models of the galaxy where we will investigate its dark matter content at small radii.

We conclude that integral field spectroscopy is indeed complementary to multi-fiber spectroscopy, and is particularly suited to studying the centers of dwarf galaxies where individual stars are resolved. We were able to extract kinematics for 17 stars with magnitudes $20<V<21$ in 14 hours of observing time on a 2.7-meter telescope. Obviously, larger telescopes with multi-object spectrographs are ideal, as slit masks are usually able to be positioned more easily. However, IFUs such as VIRUS-W can provide a low-cost alternative. 


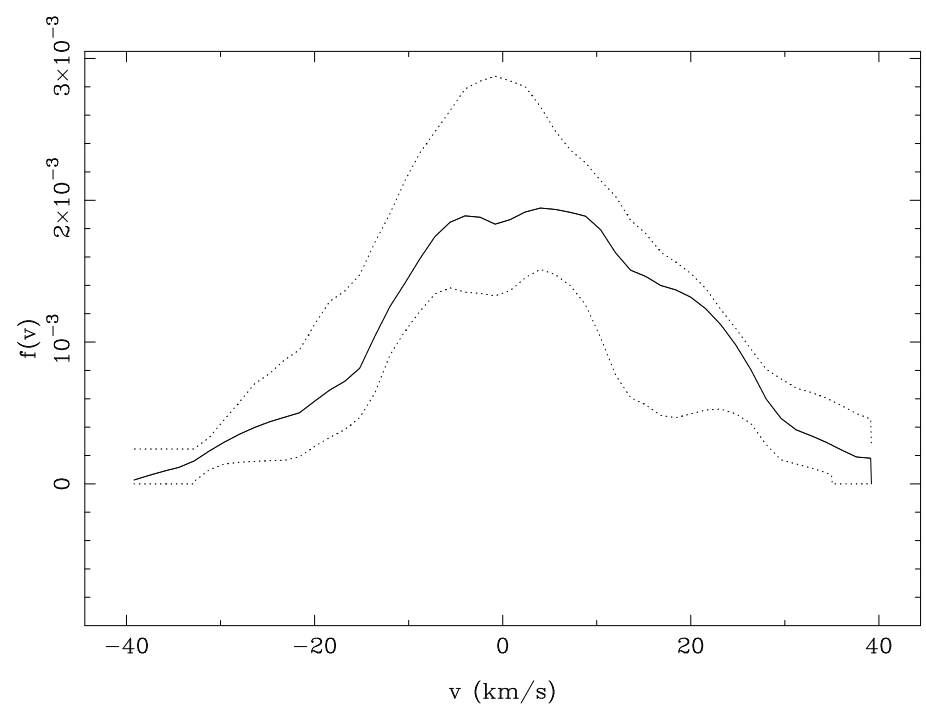

Figure 3: Line-of-sight Velocity Distribution of the 12 member stars we observe in the central region of Draco.

\section{References}

[1] Burkert, A., The Structure of Dark Matter Halos in Dwarf Galaxies, 1995, ApJL, 447, L25+

[2] Fabricius, M. H. et al., VIRUS-W: an integral field unit spectrograph dedicated to the study of spiral galaxy bulges, 2008, SPIE Proc., Vol. 7014

[3] Gebhardt, K., et al., The Centers of Early-Type Galaxies With HST. III. Non-Parametric Recovery of Stellar Luminosity Distribution, 1996, AJ, 112, 105

[4] Gebhardt, K., et al., Axisymmetric Dynamical Models of the Central Regions of Galaxies, 2003, ApJ, 583,92

[5] Jardel, J., \& Gebhardt, K., The Dark Matter Density Profile of the Fornax Dwarf, 2011, ApJ accpeted (ArXiv:1112.0319)

[6] Mateo, M. L., Dwarf Galaxies of the Local Group, 1998, ARA\&A, 36, 435

[7] Navarro, J. F., Frenk, C. S., \& White, S. D. M., The Structure of Cold Dark Matter Halos, 1996, ApJ, 462,563

[8] Persic, M., Salucci, P., \& Stel, F., The universal rotation curve of spiral galaxies - I. The dark matter connection, 1996, MNRAS, 281, 27

[9] Ségall, M., Ibata, R. A., Irwin, M. J., Martin, N. F., \& Chapman, S. 2007, Draco, a flawless dwarf galaxy, MNRAS, 375, 831

[10] Silverman, B. W., Density estimation for statistics and data analysis, Chapman and Hall, London, 1986

[11] Siopis, C., et al., A Stellar Dynamical Measurement of the Black Hole Mass in the Maser Galaxy NGC 4258, 2009, ApJ, 693, 946

[12] Walker, M. G., Mateo, M., \& Olszewski, E. W., Stellar Velocities in the Carina, Fornax, Sculptor, and Sextans dSph Galaxies: Data From the Magellan/MMFS Survey, 2009, AJ, 137, 3100 